\title{
XV.
}

\section{Epidemien der Syphilis in Franken in Folge von Operationen der Bader.}

\author{
Mitgetheilt von Dr. Lammert.
}

\begin{abstract}
$D_{\text {i }}$ e aus den Römercolonien in Deutschland bekannt gewordenen Badestuben fanden wie anderwärts so auch in Franken frühzeitig allgemeinen Eingang, und war der Gebrauch der Bäder besonders seit den Kreuzzügen im Mittelalter unter allen Volksklassen ein Lebensbedürfniss geworden. Mochte dieses besonders darin seinen Grund haben, dass ehedem der Gebrauch von Leibwäsche und deren regelmässiger Wechsel weniger allgemein war als jetzt, immerhin galt das Baden für eine heilsame diätetische Uebung, welche unter die sieben grössten Freuden jener Zeit gezählt wurde. Wer keine eigene Badstube - Stuba balnearia - im Hause hatte, besuchte wöchentlich wenigstens einmal die öffentliche Badstube, und ward diese selbst Armen, welchen bei der sonst üblichen Verpachtung der Badstuben der Genuss jener unentbehrlichen Reinigungsanstalten entzogen war, in Folge der von menschenfreundlichen Personen ausgeworfenen Legate an gewissen Tagen geöffnet (Selbäder) *). Nach der Baderordnung von Geroldshofen von 1445 mussten die beiden Bader wöchentlich vier öffentliche Bäder bereiten. Im Jahre 1557 wurden nur noch der Mittwoch und Samstag als Badetage festgesetzt. Es war jedoch nicht das Bad allein, welches die Leute dahin zog. Die Männer liessen sich Haar und Bart scheeren, die Frauen frisiren; es ward daselbst zur Ader gelassen, geschröpft und manches Ungemach kurirt. Während sich diese Baderpraxis nur auf ihre Badstube beschränkte, durften sie

*) Regest. rer, boicor. XI. 248. Eine Stiftung bestimmt: Die armen Siechen sollen alle 1/4 Tage ein Bad und $\frac{1}{2}$ Maass Wein erhalten im Hospitale zu Iphofen.
\end{abstract}


auswärts wie die Scharfrichter Verrenkungen und Brüche behandeln. Erwachsene Badegäste, sie mocbten sich schröpfen lassen oder nicht, zablten in Geroldsłofen zwei neue Pfennige, ein neunjähriges Kind einen neuen Pfennig. Das Schröpfen oder Aderlassen nach dem Bade war ein allgemeiner Gebrauch: "Arbeyt in dem mayen ist dir nicht schad, Lass zu der Adern ynd mach dir lustig bad" (Regimen sanitatis fol. 6. b. Nueremberg 1508).

Wie jedoch das Umsichgreifen des Aussatzes ehedem nicht ohne Einfluss auf den Badgebrauch blieb, indem man allentbalben åie Schwitzbäder als Schutz- und Hilfsmittel gegen jenen bedrohlichen Hautausschlag empfabl, so erlitten nun auch der Gebrauch der kunstwarmen Wasserbäder mit dem Scblusse des 15. Jahrhunderts, wo die Syphilis zur Epidemie erwuchs, einen neuen Stoss, indem sowohl das an manchen Orten übliche gemeinsame Baden beider Geschlechter, als die häufig von den ohnebin anrïchigen und unehrlichen Badern vorgenommenen blutigen Operationen zur Verbreitung der in äusserst verderblicher Weise um sich greifenden Seuche vielfach beitrugen. Nach manch traurigen Erfahrungen mieden allmälig die niederen Gesellschaftsschichten die öfentlichen Badestuben, während sich die höheren dem Gebrauche der mit dem 16. Jahrhundert in Schwung kommenden naturwarmen Quellen, "Wildbäder", zuwandten. (So fasste schon ums Jabr 1400 der bekannte Nürnberger Meistersänger und Barbier Hans Folz: "Ein puchlin von allen paten die von natur heisz sein" - ab).

Auch in Franken kam der Besuch der öffentlichen Bäder durch die allda nicht selten vorkommenden syphilitischen Ansteckungen allmälig in Verfall. Nachfolgende hierauf bezuigliche geschichtliche Beiträge liefern den Nachwois:

Der durch seine Aufzeichnungen: "All mein Richten", herausgegeben von Endter 1801, 8. - bekannte Meister Frantz, Nachrichter von Nürnberg, erzählt S. 169 Folgendes:

"1399. 13. Nov. Andreas Seitzen von*, Badersgeselle, welcher in Weisen thurn badt gearbeitet, mit seinen gesellen Michel uneins worden, ihme getrohet, ein Possen zu machen, das er an in gedenken soll, und iln darauff sein Schrepteeyssen in ein zwibel gesteckt, auch nichtern Erbes gekanet and das Eisen angeadmet; die weil nun ueber die 70 Persolnen in badt verderbet und die Frantzosen bekommen, auch die Koepfi ausgefallen, wie wol er die Frantzosen selbst 
bekommen, acht wochen im haus gelegen, hat man itm die schuldt zeben, weil aber hernaclimals er eben so wol die Leuth verderbet in andern baden, hat man ihm aus gnaden mit Ruthen ausgestrichen."

II. Die Cista medica Joa. Hornungi, Nrbg. 1626, 4. p. $423-$ theilt folgende Correspondenz mit:

1. Leonh. Doldii epistola ad S. Schnitzer. archiat. Bambergens. 1599. 17. Nov. :

"Quae de infectione per balnea refers, lubens cognovi, ut etiam supplicio affectus fuerit is, qui suspicionem dedit infectionis audivi, sed quid si nos altius penetremus, et contagii causas etiam extra balnea (quamvis per balnea longe effcaciora) quaeramus. Certe victus et aeris certa affectio, aquarum quoque, quibus lavantur corpora, huc permnltum conferunt. Sunt enim, qui neque balneatoribus usi sunt, neque qui publica balnea frequentarunt, simili lue contaminati, imo opulenti et circa victum, ut ipsis videbatur, attenti et religiosi. Praeter Libarium, qui conquestus est ea de re ante triennium, heri demum Winshemia ad me scribit medicus quidam D. Hieron. Fabritius, ultra centum diversis in locis et pagis, homines conspici sic exceptos, Quod et Weissenburgi et in pluribus oppidis etiam circa $\Lambda$ t torfium pagis et in ipso etiam oppido, plurimi jam aliquot annis, infecti hoc modo fuere et adhuc inficiuntur. Curatio non omnibus succedit, ait Chirurgus, admodum pertinacem esse morbum et pustulas aegre prorumpere (ita copiose delitescunt) et maturescere. Vale. Dabam festinanter die 17. Nor. qq."

2. Idem ad eundem: „Infectio illa balneo disseminata, mihi non parum suspecta fit ob rumores varios et malum ubique frequens in oppidis et pagis urbibusque. Nequam, qui autor putabatur, ipse infectus fuisse dicitur. Unde forte verisimele ex tua sententia (at temerariae et sordidae istac mulieres vestrum balneum inquinantes) videfur ex ipsius corporis contagio distributum per exhalationes et ealores humido mixtos vapori. Virgis coesus dimissus dicitur. Sed ulterius inquiram et diligentius perscrutabor symptomata, chirurgus, qui pracest, ait pustulas aegerrime prorumpere. Ulcera vero quae funt, valde sordida et putrida esse, cum serpendi et exedendi malitia. De coepis nunquan potui in animum inducere, ut infectio tanta oriretur, praesertim, ut ajunt, suceo imbutis sealpellis, tamen de putrescentibus coepis nil dubitarim, cum vehementer corpori obsint et etiam Galenus ad pestem ista culinaria acria corpora disponere statuat, cum et sanguinem accendant et gravem halitum spirent, caput afficiant, lethargum creent. Cum theriaca rero etiam virulentis imponi novi; de succo vero non dubito, non guod venenatus sit, sed ut rubiginem tantum inducat ferro. Succum praesentissimum ambustis cum sale praedicat remedium Paracus, et experientia testatur. An yero sordes et cum sanguine mixtus vel flamentis scalpello circumvolutis allquid aliud praestent, alias indagandun - - Norimbergae, 23. Nov. 1599.

3. Idem ad eunden: - Aliud apnd nos nuper invaluit malum, nequitia serpi balneatoris, qui ob malefacta custodiae publicae traditus est et confessus, se infinitos per sealpellum (vulgo fertur coepis infixum, Langius virulentum dicit scari- 
ficantibus allium, alii hujus halitum) infecisse in balneo, qui omnes paulo post misere ulceribus malignis, totoque corpore scabie gallica foedati, jam in domo Gallicorum separatim vivunt, ultra septuaginta homines. Symptomata omnia adeo exquisite non novi, refert tamen medicus illius nosodochii, gallicum morbum esse, cum etiam cura Gallicorum tractentar. Sed quid tibi apud Vestros hac de re notum sit, judiciumque tuum aperire te velim. Ego hactenus exquisite gallicum morbum non credidi, cum et ante biennium et ultra D. Libavius de similibus ad me scripserit plagis apud suos et vicinos populatim grassantibus, ipsis etiam balneatoribus infectis.

We is senburgo ad me scripserat medicus ante annum, totas plateas infectas esse. Conabantur passim Mercurio curare balneatores Gallicum, Libavius negaverat. Ejusdem et ego fueram sententiae et ad Luem Moravicam Th. Jordani retuli, Brunogallicum autor vocat, hac de re etiam in epistolis Cratonis cujus rei scriptum autoris exstat, quod etiam in Schenckianis observationibus lib. VII. de venenis habetur, te meminisse puto; refernntur infecti etiam qui balneum ingressi non sunt, a Th. Jordano et D. Libario. Sed vale et vive. Norimbergae, 8. Dec. 1599 .

4. Idem ad eundem. - De balneatore nescio, profecto nescio, quis magis accusandus, isne qui commisit, an qui non. Hoc statueram, voluntatem magis esse execrabilem, quam remedia illa, ad morbos eosdem efficacia, cum diversa illa pharmaca et venena, unam morborum speciem producere non potuerint, licet forte inficere quiverint. Et quod hic forte putavit sese hisce effecisse maligno animo, id alibi sponte, alibi negligentia et sordibus balneorum, alibi per infectos illatum est; sed tamen cogitabo exactius et etiam cum D. Libavio conferam. Vale. Dabam. Norimbergae satis properanter. 1602.

5. Idem ad eundem. - Gabelius refert, Mercurium succo pastinacae ejici e corpore, exemplo aurifabri sic curati - nobis tales raro tractandi sunt, cum omnis cura Gallicorum fere committatur barbitónsoribus qui sua mercuriatis peragunt - Norimbergae 1605.

III. Am 14. April 1601 erliess Markgraf Georg Friedrich zu Ansbach, "weil aus gerechtem Zorn Gottes die abscheuljche Seug und Plag der Frantzosen, der Orten, da sie vor der Zeit eingerissen, auch auf gebrauchte curam nicht nachgelassen, jetzt auch weiter um sich greife", eine Wiederholung seiner bereits vor drei Jahren, am 2. Januar 1598 ,ergangenen wolmeinenden Warnung und Befehlschreibens", des Inhaltes: „Diejenigen, so mit solcher abscheulichen Krankheit inficirt und behaftet, sollen von andern Reinen abgesondert und, gleich den andern Aussätzigen, von andrer Leute Gemeinschaft abgehalten werden, sie sollen sjch bei Hochzeiten, Gastungen, Essen und Trinken, Saigung und Nahrung der jungen Kinder, als auch in Schulen und gemeinen Badstuben, da- 
durch ihr Athem, Dunst, Schweiss, Berührung, Lager, Sitzstetten, Lassköpfe und Eisen andere Gesunde in merklicher Unzahl vergiftet haben, nicht gebrauchen lassen. Inficirte Kinder sollen weder von gesunden Personen gesängt, noch zum Schulbesuche gelassen werden. Schulmeister und Bader werden ernstlich und bei hohen Strafen aufgefordert, angesteckte Personen weder in Schulen noch in Bäder aufzunehmen; die Obrigkeiten der Städte und Dörfer werden daneben aufmerksam gemacht, fleissige Vermahnung und Bestallung $\mathrm{zu}$ thun, dass unerfahrenen Balbirern, Badern und alten Weibern, welche solche arme inficirte Leute mit Quecksilber, gesottenem Zinnober und andern dergleichen giftigen Sachen behandeln, ihre schädliche Kunst niedergelegt, und hergegen erfahrene Aerzte und ordentliche curae gebraucht werden mögen". Für die armen Kranken wird endlich eine Kollekte im Fürstenthum bewilligt.

IV. Im Jahre 1603 verbreitete sich in Bamberg die Lustseuche und wurde der dortige Bader, Leonhard Kurmreutter, als Urheber des Uebels beschuldigt. Seine hierauf beziigliche Confessio lautet nach Gregor. Horstii, op. med. II. 431 also:

„Er hat drey oder vier Wochen lang das Mückenpulver auff den Lederfeilen gestrichen und die Lasseisen darauff gewetzt.

Er hat in ein Grillen die Schröpffeisen gerieben, und den Leuten, die inficirt worden, damit geschröpfft, darauff ihnen das Blut verstanden, und auff den Schröpffmahlen, Rufen und Beulen auffgeschossen.

Er hat die Krotten gedörrt und das Pulver in einen Zuber mit Wasser gethan, das Wasser auffgerührt und auf den Badófen gegossen, daraus ein Dampff und.Dunst erweckt worden".

Diese Dinge machten den Aerzten viele Bedenken, wie dies aus Greg. Horst. opp. med. $431-433$ erhellt. Daselbst findet sich Observatio de malitiosa scarificatione Epist. Sigismund. Schnitzeri, archiatri Bambergensis ad Andream Libavium, 1603, 2. Febr.:

"De ista Balneatoris venefici confessione quid sentis? Infecti scatebant pustulis et ulceribus, quasi cancrosis, non tantum in 'locis scarificatis, sed toto fere corpore, patiebanturque artuum dolores immensos, tanta debilitate, ut nisi suffulti, lecto exire non potuerint. Horum qui plurimi erant, aliqui curati sunt ligno decocto et alexipharmacis, caeteri magnitudine mali occubuerunt. De pul- 
vere myoctono et bufonibus pulverisatis ne quid dicam, saltem grylli quit virium ad haec mala contulerint, ex te audire liheret etc."

Epistol. Andr. Libavii ad S. Schnitzerum, dat. Rotemburgi, 2. Mart. 1603. "Grylli nostrates quinam sint, non constat inter omnes. Haec est illa blatta betrusca Mathiol. (gem. Küchenschabe), cumque in nostris balneis et pistrinis frequens sit, tenebrasque amet, quid obstat, quin dicamus eliam gryllum esse aliquando dictam blattam - - cum sanguis in scarificatione fluere desiit, veneno seu sanie gryllorum delibuto phlebotomo, considera, an non tota natura adversetur sanguinis vitae, jugulefque vel mutet suo veneno spiritum et calorem nativum in sanguine, - tanquam in morsu canis rabidi - corruptio exoritur ete."

Epist. Schnitzeri ad Libavium 1613. 13. Aug. (Horst. II. 484): „Infra scriptum balneatorem rotae impositum aves per multas septimanas non attigerunt, licet cnim balneator usus fucrit antidoto, diuturnus tamen corporis venenati usus praevaluit."

Libarii responsum ad Schnifzerum, 1. Sept. 1613 Coburgo datum: „Balneaforem illum veneficum rolae impositum a bavibus intactum mansisse, suspicor magum quoque fuisse (Qni in vicinia nostra in cruce pependit, idem a corvis fuit immunis per aliquot dies). Manes ejus, ni velis factores acres, practeracta armenta tumultuari fecerunt."

V. Aus den handschriftlichen, im histor. Vereine von Unterfranken und Aschaffenburg (sub F. 137) niedergelegten Acten, die Klage der Gemeinde Sommerach am Main gegen den Bader Bernhard Holzheuser, "in specie 13 inficirte Weibss undt 1 Mansspersohn, auch dess Baders Von der Gemeind Beschuldtigten Unfleiss, Uebermuth undt Ungehorsamb betr." - ersehen wir unter dem 18. Mai und 13. Juni 1615, dass am Schlusse des verwichenen und Anfange dieses Jahres die Syphilis in Sommerach um sich griff, und wird gegen den Bader daselbst als den Urheber des Uebels wegen seines, muthwillens, fräuelss, trutzen undt anderer Ungebür" Beschwerde erboben.

„Nur befindet sichs, dass Innerhalb wenig monaten ezliche Personen mit der seucht, so man insgemein morbum galicum nent, inficirt darunter 11 weibss undt 1 manspersoln, so in Gemeindtbad mit schrepfen also verlezt, dass auff den Köpfen die Blattern sich eraigt, hernacher denselben die Arm nndt andern Glidter also schwer undt schwach worden, dass sie sich in äie Chur begeben müssen." In der Rechtfertigung des Baders vom 15. Juni bringt er u. A. vor: „Belangendt dann die hauptsach und der inficirten persobnen gethane Klagh undt aussagh, wie wol ich dieselben in effectu und insgemein bei allen dahin gericht befindt, dass sy by dem baden schrepfen, weil Inen die Köpf und Hieb geschworen wnd dardurch die abschenlich seucht sich mehr und mehr eraignet, und aussbrüchig worden, die schuldt und ursach gern zumessen wolten; so ist aber hiegegen und Dem zu- 
wider, in dieser gantzen refier landts $Z$ u franken ob und unterhalb Steigerwallslandikündig, das fleider von $z$ wantzig jaren hero diess abscheuliche Kranckheit an viel underschiedtlichen orten, alss Kitzingen, Grossen- unnd Kleinlanckbeim, Wiesenbrun, Wiesentheit, Rüdenhausen, Abbtschwindt, Geiselwindt und viel andere mebr orten auss sonderbaren wolverschuldten straff Gottes und unsers bösen unordentlichen wessens und lebens halben weit eingerissen, und drüber so ein vielfeltige gemeine Klagh, dergleichen bei unsern frommen alten Voreltern so gemein nie gewesen, gehört worden" u. s.f. Die von Fürstbischof Julius zur Untersuchung der Sachlage nach Sommerach gesandten Leibmedici und Wundärzte Georg Beier und Georg Hübner vernahmen daselbst am Freitag nach Corporis Christi (8. Juni) beide Parteien und legten nach Untersuchung der Kranken das Zeugniss ab, dass der Bader die Schuld der Ansteckung nicht 1rage, sondern dass dos im Körper verborgene Gift durch Anwendung der Schröpfköpfe blos Luft bekommen und einen Ausgang gefunden habe; ebenso glaubten sie auch die Gescbwöre auf dem kopfe ron den beim Haarscheeren zufällig entstandenen. kleinen Scbnitten ableiten zu müssen. - Auf Grund der geplogenen Untersuchung erliess jedoch am 16. Dezember d. J. Fürstbischof Julius den Bescheid, dass „mehrbenannter Bader entweder die Badstuben verkauffen, oder aber im Flecken Sommerach des Badbaltens, Curiren und Verbindens sich zumal entschlagen, und den Unterthanen freystehen soll, Ihres gefallens die Bad $z u$ besuchen und ein Bader, wo sie wollen, zu gebrauchen."

VI. Unter dem 23. März 1624 theilt Dr. Marcus Widemann, Physicus zu Ysny, folgenden Bericht dem D. Gr. Horstius (opp. med. Il. 433) mit:

"Dr. abr. Boxbarter, phys. Windsheimensis hisce diebus ad me scripsit: „At nunc, ecce, inquit, grassatur apud nos inter cives passim lues quaedam epidemica contagiosa, qua ultra 70 homines jam infecti sunt. Symptomata eadem plane sunt, quae luis Moravicae a Th. Jordano quondam descripta*). Nam a scarificatione omnes boc malum contraxere, pustulis primum in cucurbitularum sedibus abortis lividis, latis, crustaceis, exedentibus, dolentibus quibusdam, nonnullis vero indolentibus. Has seguuntur lassitudo tolius corporis, articulorum dolores, cephalalgiae nocturnac, ulcera magna plagadaenica, gummata, dolores osteocopi, tubercula, oris inflammationes et exulcerationes, alque summiatim, quae morbum gallicum comitari solent, fere omnia. Rogatus ego a senatu nostro, quaenam bujus mali causa esset, contagium per contactum et fomitem esse affirmavi, cum praeter hos, qui scarificatione usi sunt, nemo fuerit infectus, iisque solum, quibus in eodem balneo sanguis extractus, - in altero balneo fere

*) Boxbarter irrt, wenn er die Symptome der von ihm gut gezeichneten Lues identisch lält mit jenen, welche Thom. Jordanus in seinem, „Pestis Phainomena, Frkft. 1576, von der im J. 1566 ïber fast ganz Europa verbreiteten sog. Ungarischen (Mährischen) Krankineit niederlegte. Letztere ist wahrscheinlich ein ächter Petechialtyphus, welchem namentlich, da ihn gleichzeitig eine unerhörte Theurung begleitete, die ärmere Volksklasse zum Opfer fiel. 
nemo fuerit infectus. Potest enim scalpellum vel malitiose intoxicari, vel, cum tam inhumanum facinus in neminem cadere existimem, cum ab infecto ad sanum balneator transiit, eodemque scalpello, virulenta labe adhuc sordido, ipsum vulneravit, illius sanguinem per contagium corrumpi, qui postquam corruptus fuerit, alios etiam potuit inficere, contagio in infinitum sic serpente etc."

Idem ad Horstium, 17. December 1627: „De Boxbateri methodo cur. nihil habeo dicere, cum ante biennium ex hac vita excesserit - originem harum infectionum indaganti, haec niht causa sese offert, quod scilicet, scalpella, quibuis balneator ad scarificationem usus fuit, infecta fuerint sanguine alicujus morbo gallico laborantis - Windshemii ne suspicio quidem ulla veneficti orta fuit etc."

Wie in Franken, so kam auch anderwärts dieselbe Verbreitungsweise der Syphilis vor und sei noch scbliesslich erwähnt, dass sie (nach Horst. opp. med. II. 319) im Jahre 1622 in Uim auftrat: "Lues gallica Ulmae post scarificationem in balneo publico contracta". Hiefür suchte unser Autor keine Begründung in der Beruhrung mit Zwiebelsaft, oder Mäuse-, Rröteñ- und Schwabenpulver, sondern spricbt sich p. 434 vernünftiger dahin aus: „Hane infectionem textoris absque malitia, casu potius, quod instrumenta scarificationis cuidam lue affecto fortassis inscienter adhibita fuerint, subsecutam facile credo".

\section{XVI. \\ Ueber die plysiologische Wirkung des Bebeerins und llicins.}

Von Prof. J. F. H. Albers in Bonn.

Ws ist nicht ohne Werth für den practischen Arzt, sich die Frage zu beantworten, worin wohl die gemeinsame physiologische Wirkung begründet sei, welche der therapeutischen Anwendung jener Mittel zu Grunde liegen mag, die gegen ein und dasselbe Leiden mit Erfolg in Anwendung gebracht sind. Es wird aus einem Vergleich der physiologischen Wirkung solcher Mittel ersichtlich, welche 